\title{
Donación de órganos y trasplante en la era COVID-19: ¿Realmente se deben parar los programas?
}

\author{
Organ Donation and Transplantation in the COVID-19 era: \\ Should programs really stop?
}

\author{
Roger Martin', Melibea Sierra-Ruiz², Luis M. Barrera-Lozano³ \\ 1 Cirujano General Universidad Nacional de Colombia. Fellow Cirugía de Trasplante de Órganos Abdominales Universidad ICESI, \\ Unidad Funcional de Trasplantes Fundación Valle del Lili, Cali, Colombia. \\ 2 Research Fellow, Harvard University, Boston, MA, USA. Centro de Investigaciones clínicas (CIC), Fundación Valle del Lili, Cali, \\ Colombia. \\ 3 Cirujano de Trasplante de Órganos Abdominales, Universidad de Antioquia. Servicio de Cirugía Biliohepatopancreática, Trasplantes \\ y Rehabilitación Intestinal, Hospital San Vicente Fundación. Rionegro, Colombia.
}

\section{Resumen}

Durante los primeros meses de la pandemia por SARS-CoV2 (Coronavirus 2 del Síndrome Respiratorio Agudo y Grave), el agente etiológico de la Enfermedad Infecciosa por Coronavirus de 2019 (COVID-I9), la actividad de donación y trasplante de órganos en todo el mundo se ha visto claramente afectada. Las principales razones que en este momento motivan el cese parcial o total de los trasplantes son: I) la carga asistencial que genera el manejo de un potencial donante en la Unidad de Cuidado Intensivo (UCI), 2) el alto riesgo de contagio entre donante y receptor, 3) el riesgo de inmunosuprimir a un paciente en medio de la pandemia y 4) la escases de camas de UCI.

A pesar de que el mundo está enfrentando a una enfermedad emergente que merece especial atención, al mismo tiempo continúan prevaleciendo las complicaciones asociadas a las demás enfermedades, incluyendo las complicaciones de patologías crónicas en estado terminal. La decisión de continuar con los programas de trasplante se debe basar en el comportamiento local del virus y en la capacidad asistencial de cada una de las instituciones. En Colombia, el comportamiento epidemiológico del SARS-CoV2 varía significativamente entre las diferentes regionales, permitiendo a las instituciones que hasta el momento presentan poca carga de atención del COVID-I9 retomar sus actividades de trasplante. De esta manera se propone un balance entre mantener las medidas de prevención y atención del COVID-I9 y continuar ofreciendo los servicios de trasplante, principalmente a los pacientes con alto riesgo de morbi-mortalidad en lista de espera.

Palabras clave: COVID-I9; virus del SRAS; coronavirus; infecciones por coronavirus; pandemias; cirugía general; trasplante.

Fecha de recibido: 12/04/2020 - Fecha de aceptación: 13/04/2020

Correspondencia: Roger Martin G. Dirección: Unidad funcional de trasplantes, Fundación Valle del Lili. Carrera 98 \# 18-49, Torre 6 Piso 5. Cali, Colombia. Teléfono: 314-4130607

Correo electrónico: jrmarting@unal.edu.co

Citar como: Martin R, Sierra-Ruiz M, Barrera-Lozano LM. Donación de órganos y trasplante en la era COVID-19: ¿Realmente se deben parar los programas? Rev Colomb Cir. 2020;35:227-34 / Especial COVID-19. https://doi.org/10.30944/20117582.624

Este es un artículo de acceso abierto bajo una Licencia Creative Commons - BY-NC-ND https://creativecommons.org/licenses/by-ncnd/4.0/deed.es 


\begin{abstract}
The SARS-CoV2 (Severe Acute Respiratory Syndrome-related to Coronavirus 2) pandemic, which is the etiological agent of the Coronavirus Infectious Disease 2019 (COVID-I9), organ donation and transplantation activity throughout the world has been clearly affected. The main reasons that currently motivate the partial or total cessation of transplants are: I) the burden of care burden generated by the management of a potential donor in the Intensive Care Unit (ICU), 2) the high risk of donor/recipient viral transmission, 3) the risk of using immunosuppressing a patient in the midst of the pandemic, and 4) the shortage of ICU beds.

Despite the fact that the world is facing an emerging disease that deserves special attention, at the same time the complications associated with other diseases continue to prevail, including complications of end-stage chronic diseases. The decision to continue with the transplant programs should be based on the local behavior of the virus and the healthcare capacity of each of the institutions. In Colombia, the epidemiological behavior of SARS-CoV2 varies significantly between different regions, allowing institutions that, until now, have little burden of attention from COVID-I9, to resume their transplant activities. In this way, a balance is proposed between maintaining the prevention and care measures of COVID-I9 and continuing to offer transplant services mainly to patients with a high risk of morbidity and mortality on the waiting list.
\end{abstract}

Keywords: COVID-I9; SARS virus; coronavirus; coronavirus infections; pandemics; general surgery; transplantation.

\section{Introducción}

El trasplante de órganos sólidos se ha establecido durante los últimos 50 años, como la mejor opción de tratamiento para múltiples enfermedades que conllevan a la falla irreversible de uno o varios órganos. Hoy en día es considerado un tratamiento "salvador de vidas" que ha mostrado muy buenos resultados a corto, mediano y largo plazo ${ }^{\mathrm{I}}$. Por tratarse de una práctica que involucra el uso de medicamentos inmunosupresores, una de las amenazas constantes son las infecciones oportunistas que pueden afectar a los pacientes trasplantados, especialmente en el postrasplante temprano (antes de seis meses).

Entre las infecciones oportunistas merecen especial atención los virus emergentes, ya que al ser agentes novedosos, es difícil predecir el comportamiento que tendrán al infectar a un paciente inmunosuprimido. Desde los años 8o, se han presentado varios brotes con virus emergentes y todos en su momento han generado gran preocupación entre la comunidad médica y científica que atiende pacientes con trasplantes. Algunos ejemplos son: el Virus de Inmunodeficiencia Humana (VIH), a finales de los 80 e inicio de los 90; el Virus del Nilo Occidental, que emergió en los Estados Unidos hacia finales de los
90; el SARS-CoV en 2002-2003; la pandemia por AHINI en 2009; el MERS-CoV en 20I2; el Zika; el Ébola y ahora el más reciente SARS-CoV2 desde finales de $2019^{2-5}$. Todos ellos han obligado a los centros de trasplantes a generar estrategias para disminuir el riesgo de trasmisión entre donante y receptor, para minimizar el riesgo de infección postrasplante entre los nuevos receptores y para optimizar el manejo de los recursos físicos y humanos en salud, disminuyendo la carga asistencial en medio de cada una de estas crisis ${ }^{3,6}$.

La mayoría de estos brotes se limitaron a zonas geográficas específicas y se pudieron manejar sin generar un impacto negativo considerable en la actividad de donación y trasplante a nivel mundial. Sin embargo, esta nueva pandemia por SARS-CoV2 (Coronavirus 2 del Síndrome Respiratorio Agudo y Grave), agente etiológico de la Enfermedad Infecciosa por Coronavirus de 2019 (COVID-I9), ha mostrado un comportamiento epidemiológico sin precedentes, dada su rápida expansión, sus mecanismos de transmisibilidad y la gran carga asistencial que genera sobre los sistemas de salud. Por lo anterior, muchos de los grupos de trasplante en los países en fase de mitigación con circulación libre del virus han decidido parar sus programas. 
Ha pasado poco más de un mes desde que la Organización Mundial de la Salud (OMS) hiciera un llamado a un estado de emergencia de salud pública de importancia internacional y declarara la pandemia por COVID-I9 el II de marzo de 2020 7. Muchas han sido las estrategias implementadas alrededor del mundo para disminuir la tasa de contagio y optimizar el manejo de los pacientes infectados. Sin embargo, el número básico de reproducibilidad, también llamado R-zero (Ro) ó R-Naught parece no bajar. Este número representa la cantidad de personas que en promedio serían infectadas por un paciente COVID-I9 positivo; los reportes actuales en zonas geográficas de alta transmisibilidad estiman Ro $>3$ [2.22-3.58] lo que indica que un paciente infectado puede contagiar a aproximadamente tres personas más y la pandemia seguirá su crecimiento exponencial ${ }^{8,9}$. Colombia, según la OMS, continúa estando fuera de la lista de los países con alta transmisibilidad, ocupando al momento de realizar esta revisión [Io de abril de 2020], la posición número 46 en el mundo y el quinto lugar en la región según el número de casos nuevos para COVID-I9 ${ }^{\mathrm{I}}$.

A pesar de tener un Ro más bajo en comparación con países que han sido epicentro de la pandemia, de manera preventiva algunos programas de trasplante en el país han decidido parar completamente sus actividades, mientras que otros han venido trabajando a media marcha, en concordancia con las políticas nacionales y con las recomendaciones internacionales. Lo que motiva esta iniciativa es la necesidad de liberar camas en las Unidades de Cuidado Intensivo (UCI), minimizar la carga asistencial que genera el cuidado de un potencial donante, disminuir la estancia hospitalaria de pacientes inmunosuprimidos que, al parecer, presentan un mayor riesgo de contagio, y evitar la transmisibilidad de SARS-CoV2 del donante al receptor.

A pesar de que esto es muy positivo, no se debe perder de vista la realidad de que durante la pandemia por COVID-I9 continúan prevaleciendo todas las demás enfermedades que ya existían, entre las que se incluyen aquellas que requieren un trasplante de órgano como medida salvadora de vida. Aunque varios autores proponen que no es urgente seguir trasplantando y que los programas se podrían parar por varios meses sin afectar la morbi-mortalidad de las personas en lista de espera, esto no es del todo cierto; por ejemplo, se estima que la supervivencia a 3 meses en lista de espera de trasplante de hígado es del $7 \mathrm{I}, 2 \%$ en comparación con una supervivencia del $94 \%$ entre los pacientes que se trasplantan. El riesgo de mortalidad a 3 meses aumenta en un $58 \%$ entre los pacientes que tienen un MELD Score $>30 \mathrm{y}$ presentan encefalopatía como complicación de la cirrosis ${ }^{\mathrm{II}, \mathrm{I} 2}$.

Así las cosas, se considera pertinente analizar, de manera rigurosa, la información hasta el momento recopilada, con el fin de plantear la posibilidad de nuevas estrategias que permitan un balance para continuar realizando trasplantes, sin descuidar las medidas de prevención y atención del COVID-I9 en cada institución. La información se presentará en tres preguntas importantes, correlacionadas entre ellas, cuyas respuestas pretenden aportar un poco más al conocimiento del tema.

¿Se podrían continuar los trasplantes de órganos sólidos en Colombia durante la pandemia por COVID-I9?

En el contexto de una pandemia es importante evitar las especulaciones y suposiciones. Antes de disponer cualquier medida, se deben estudiar los hechos reales y las cifras epidemiológicas que, aunque a veces sesgadas por el sub-registro, son la única fuente de información que orienta la toma de decisiones. $\mathrm{Al}$ momento de definir si se para o se continúa con un programa de trasplantes en la era COVID-I9 son muchos los interrogantes que se ponen sobre la mesa. La respuesta a la mayoría de éstos es incierta y varía de una región a otra, dependiendo del comportamiento epidemiológico del virus; lo que es cierto para un país, puede que no lo sea para otro; las medidas adoptadas en una región, puede que no sirvan en otra.

Por lo tanto, es muy difícil tomar decisiones globales y adoptarlas de la misma manera en to- 
das las instituciones con programas de trasplantes en el mundo, obligando a tomar decisiones independientes de manera regional. La justificación a la premisa anterior, se basa en el hecho de que aunque la pandemia por SARS-CoV2 ya ha alcanzado a I83 países, la penetrancia de la enfermedad no ha sido la misma. Incluso, el Ro y la tasa de mortalidad (tasa de fatalidad) varían significativamente entre regiones del mismo país. Podríamos ilustrar lo anterior con dos ejemplos: en China, Wuhan y toda la región de Hubei fue la más afectada desde el inicio de la pandemia, mientras que otras regiones del mismo país presentaron un número de contagios mucho menor ${ }^{13}$. Así mismo ocurrió en Italia durante el mes de marzo de 2020, donde Lombardía, ubicada al noroccidente, ha sido la región más afectada, convirtiéndose en el epicentro de la pandemia en Europa; mientras que en el suroriente Italiano, el SARS-CoV2 ha presentado un comportamiento mucho más inocuo ${ }^{14}$.

Por consiguiente, las decisiones preventivas y de manejo del COVID-I9 no deben ser iguales para todo el mundo y pueden cambiar entre las diferentes regiones de un país. Por supuesto, hay decisiones que sí son de importancia internacional, pero otras podrían variar según el comportamiento epidemiológico en cada región. Específicamente hablando del tema en desarrollo, la decisión de parar de manera preventiva los programas de donación y trasplante en toda Colombia, en un momento donde el comportamiento epidemiológico del COVID-I9 no es igual en todas las regiones y donde la carga de la enfermedad ocupa menos de io \% de la capacidad de respuesta hospitalaria, es un tema que podría debatirse e invita a la toma de decisiones individuales entres las diferentes regionales de trasplante del país.

En esta pandemia, Colombia continúa estando fuera de la lista de los países con alta tasa de transmisibilidad según la OMS ${ }^{15}$. Al momento de realizar esta revisión se habían reportado 2473 casos positivos para COVID-I9 en todo el país, 40,I \% de estos casos registrados en Bogotá, con
80 muertes relacionadas a nivel nacional (tasa de mortalidad de 3,2\%) ${ }^{\text {Io }}$. Estas son unas cifras alentadoras, que permitirían, hasta el momento, contemplar la posibilidad de continuar con los programas de trasplante de órganos sólidos, al menos en algunas regionales.

Por citar casos de países que han continuado trasplantando, podríamos hablar de Italia, España y los Estados Unidos, lugares donde con un mayor número de contagios de SARS-CoV2, una mayor ocupación hospitalaria e incluso un mayor desgaste en los sistemas de salud por la pandemia, han continuado con los programas de trasplante, especialmente en las regiones menos afectadas de cada país ${ }^{16,17}$. Haciendo la salvedad de las diferencias entre los sistemas de salud de los países antes mencionados y el nuestro, en Colombia podría seguirse el mismo ejemplo si un centro trasplantador dado decide hacerlo. Podría ser el caso de grandes instituciones ubicadas en regiones del país con baja tasa de contagio por SARS-CoV2 y que además cuentan con un número suficiente de camas de UCI, que hasta el momento se están utilizando en menos del IO\% para casos COVID-I9 positivos.

Aunque este porcentaje puede cambiar en las próximas semanas, mientras esto ocurre, estas instituciones podrían habilitar espacios y rutas libres de COVID-I9 (o COVID-I9 free pathways) para continuar con los programas de trasplante de órganos sólidos, minimizando el riesgo de contagio intrahospitalario y con una mínima ocupación de camas en UCI. Esta es una posibilidad viable que se debe discutir de manera individual en cada una de las instituciones que realizan trasplantes. Para ayudar a la toma de decisiones, se podría seguir el esquema planteado por Paolo Grossi y Deepali Kumar de la Unidad de Trasplantes y Cirugía Hepatobiliar del Departamento de Ciencias Quirúrgicas de la Universidad de Roma, quienes proponen continuar con la actividad de donación y trasplante en un 25, 50, 75 o incluso Ioo \% según la capacidad de cada institución y basados en el comportamiento del virus en cada región ${ }^{16,17}$. 
¿Cómo se podría disminuir el riesgo de transmisión de SARS-CoV2 entre donante y receptor?

Uno de los retos de continuar con la actividad de donación y trasplantes en la era COVID-I9 es el hecho de enfrentarse al riesgo de transmisión vertical del virus entre donante y receptor. Aunque nunca se ha reportado el caso de transmisión de SARS-CoV2 asociado al trasplante (ni de SARS-CoV ni de MERS-CoV), existe un riesgo real, el cual no sólo debe ser estudiado sino que además debe ser contemplado y plasmado de forma clara en los consentimientos informados previo al trasplante. Teniendo en cuenta que en Colombia el $80 \%$ de los trasplantes se realizan con órganos de donante cadavérico en muerte cerebral (INS 20I9), durante el cuidado de estos potenciales donantes se debe promover la implementación de todas las medidas de prevención de COVID-I9 establecidas de forma institucional para minimizar el riesgo de contagio en UCI.

Siguiendo los lineamientos de la OMS para disminuir el riesgo de transmisión vertical entre donante y recetor, a todos los potenciales donantes en muerte cerebral se les debe realizar la prueba para SARS-CoV2 por RT-PCR ${ }^{18}$. La muestra debe ser tomada de lavado broncoalveolar (sensibilidad del 93\%) o de hisopado nasofaríngeo (sensibilidad del $63 \%)^{19}$. Esta prueba amplifica en tiempo real el RNA de tres genes específicos presentes en el virus (gen N, gen E y gen de la transcriptasa reversa) ${ }^{19}$. Si la prueba es positiva, se debe descartar el donante ya que entre un $\mathrm{I}^{-2}-2 \mathrm{O} \%$ de los casos positivos para COVID-I9 hacen viremia, aumentando el riesgo de la presencia del virus en órganos extrapulmonares ${ }^{20,21}$. Adicionalmente, se debe recordar el tropismo que tiene el SARS-CoV2 por la enzima convertidora de angiotensina 2 (ACE2), la cual no sólo se encuentra en el pulmón, sino que en su estado no soluble también se encuentra anclada a la superficie del epitelio renal, aumentando así la posibilidad de que en los riñones tomados de donantes COVID-I9 positivos se encuentren células epiteliales con inclusiones virales por SARS$\mathrm{CoV}{ }^{22}$. También se deben descartar los donantes si existe una alta sospecha clínica e imagenológica de neumonía, aunque la prueba por RT-PCR arroje resultados negativos. No se debe realizar una prueba rápida de detección de anticuerpos por serología, ya que un resultado negativo no descarta la presencia de la infección ${ }^{23}$.

A los donantes vivos, que en Colombia aportan entre un 20-25\% de los órganos para trasplante, también se les debe realizar la prueba para SARS-CoV2 por RT-PCR, especialmente si vienen de regiones del país con alta tasa de transmisibilidad del virus. La muestra igualmente debe ser tomada a través de hisopado nasofaríngeo ${ }^{18}$. Aunque la mayoría de grupos en el mundo han optado por diferir los trasplantes con donante vivo, si en algún momento durante la pandemia y como medida salvadora de vida, es necesario hacer un trasplante con donante vivo, se les debe aclarar a los donantes que el riesgo de contagio de SARS-CoV2 durante la donación es mayor al riesgo de contagio en la comunidad; lo anterior relacionado con la instrumentación de la vía aérea y con la estancia en un ambiente hospitalario ${ }^{24}$.

Con relación a los receptores, que la mayoría son pacientes en lista de espera para trasplante, recae sobre cada grupo tomar la decisión de cuándo hacer la prueba para COVID-I9. Las guías italianas, por ejemplo, recomiendan evaluar al receptor con prueba molecular por RT-PCR y con prueba rápida de detección de anticuerpos cuando haya presentado un cuadro clínico que sugiera neumonía por SARS-CoV2, así ya esté resuelto ${ }^{25}$. Si la realización de la prueba prolonga el tiempo de isquemia fría, debería optarse por otro receptor. Es importante tener en cuenta que un gran porcentaje de los posibles receptores de un trasplante de riñón son pacientes con terapia de reemplazo renal tipo hemodiálisis, lo que no sólo aumenta el riesgo de contagio de SARS-CoV2 previo al trasplante, sino que además es un factor de riesgo de severidad durante el COVID-I9 ${ }^{26}$.

Así las cosas, las estrategias para disminuir el riesgo de transmisión del SARS-CoV2 entre donante y receptor que hasta el momento han sido implementadas por los grupos de trasplante que mantienen su actividad durante la pandemia, se enfocan principalmente en prevenir el riesgo 
de contagio de los potenciales donantes en UCI y en evaluar por RT-PCR para COVID-I9, tanto al donante como al receptor, según lo descrito anteriormente.

¿Qué pasa con el alto riesgo de morbi-mortalidad por COVID-I9 asociado a los protocolos de inducción en el postrasplante inmediato?

Una de las principales razones para parar los programas de trasplante de órgano sólido durante la pandemia por COVID-I9, ha sido el riesgo que se corre al exponer a un paciente agudamente inmunosuprimido a la posibilidad de adquirir una infección por SARS-CoV2. Más allá de la utilización de camas de UCI ó del riesgo de contagio entre donante y receptor, existe una mayor preocupación por la morbi-mortalidad asociada al hecho de inmunosuprimir de novo al receptor de un trasplante en un momento histórico en el que hay un alto riesgo de COVID-I9.

Es muy diferente la situación con los pacientes en pos-trasplante tardío, que reciben medicamentos inmunosupresores de mantenimiento de forma crónica, en los cuales no existe un riesgo de contagio y/o severidad del COVID-I9 superior al de la población general ${ }^{27}$. Pero en este caso, y hablando del pos-trasplante temprano, existe una preocupación especial en los pacientes recién trasplantados, específicamente por el uso de medicamentos depletores de linfocitos $T$ durante la inducción, como la Timoglobulina ${ }^{\circledR}$. Hasta el momento no hay reportes en la evidencia científica que describan cuál sería el comportamiento clínico del COVID-ı en un paciente que haya recibido Timoglobulina ${ }^{\circledR}$, pero se considera que el uso de este medicamento sí constituye un factor de riesgo de severidad ${ }^{28}$.

De esta manera, los grupos que decidan continuar con los programas de trasplante renal durante la pandemia, podrían utilizar la estrategia de seleccionar receptores con bajo riesgo inmunológico y con una alta histocompatibilidad HLA, con el fin de poder omitir el uso de Timoglobulina ${ }^{\circledR}$ y optar por protocolos de inducción únicamente con altas dosis de esteroides, seguidos de un inicio temprano de la inmunosupresión de mantenimiento.
Este no es el caso en el trasplante hepático, ya que usualmente no se utilizan depletores de linfocitos $\mathrm{T}$ durante la inducción en pacientes receptores de hígado.

Además de omitir el uso de Timoglobulina ${ }^{\circledR}$, con la ayuda de los diferentes comités epidemiológicos y de seguridad del paciente, se debe evaluar en cada institución la posibilidad de crear una ruta libre de COVID-I9, desde la recepción del paciente previo al trasplante, hasta su último día de estancia hospitalaria, debe permanecer en un espacio libre de COVID-I9. Asimismo, se deben implementar protocolos tipo "fast-track" para disminuir el tiempo de estancia en UCI y el tiempo total de hospitalización postrasplante.

$\mathrm{Si}$ a pesar de esto se sospecha que un paciente recién trasplantado se ha contagiado de SARS$\mathrm{CoV} 2$, se debe confirmar su diagnóstico teniendo en mente el hecho de que en los estados de inmunosupresión severa aumenta la probabilidad de tener un falso negativo, incluso en las pruebas moleculares por RT-PCR ${ }^{17}$. De confirmarse la infección, se debe proceder según los esquemas de tratamiento institucionales teniendo presente la interacción medicamentosa ${ }^{29-31}$. Aunque todas estas nuevas consideraciones no son tarea fácil, pueden ser una solución para los centros que, durante la pandemia, contemplen la posibilidad de continuar trasplantando sin afectar la seguridad del paciente.

\section{Palabras finales}

No es fácil la toma de decisiones en este momento de crisis sanitaria por la que atraviesa el mundo; sin embargo, se debe reconocer que la situación particular del país, hasta el momento, permite la posibilidad de continuar con las actividades de donación y trasplante, especialmente en algunas regionales del país en donde existen instituciones con una infraestructura suficiente para generar nuevas estrategias y superar las barreras que ha traído esta nueva pandemia. La decisión de continuar o no con los trasplantes se debe evaluar de manera rigurosa buscando, en la medida de lo posible, un balance entre mantener los protocolos de prevención del COVID-I9 
y continuar ofreciendo el trasplante de órganos sólidos como tratamiento salvador de vida, especialmente a los pacientes en lista de espera con alto riesgo de mortalidad.

\section{Cumplimiento de normas éticas}

Consentimiento informado: Esta publicación es una revisión de la literatura, y como tal no hay necesidad de un consentimiento informado ni de aprobación del Comité de Ética Institucional.

Declaración de conflicto de intereses: Los autores no declaran ningún conflicto de interés.

Fuentes de financiación: Recursos propios de los autores.

\section{Referencias}

I. Abecassis M, Bridges ND, Clancy CJ, Dew MA, Eldadah B, Englesbe MJ, et al. Solid-organ transplantation in older adults: current status and future research. Am J Transplant. 20I2;12:2608-22. https:doi. org/IO.IIII/j.I600-6I43.20I2.

2. Blumberg EA, Fishman JA. Zika Virus in transplantation: Emerging infection and opportunities. Am J Transplant. 20I7;17:599-600. https:doi.org/IO.IIII/ ajt.I4083

3. Kaul DR, Mehta AK, Wolfe CR, Blumberg E, Green M. Ebola virus disease: implications for solid organ transplantation. Am J Transplant. 2015;15:5-6. https:doi. org/IO.IIII/ajt.I3093

4. Kumar D, Tellier R, Draker R, Levy G, Humar A. Severe Acute Respiratory Syndrome (SARS) in a liver transplant recipient and guidelines for donor SARS screening. Am J Transplant. 2003;3:977-8I.

5. Levi ME, Kumar D, Green M, Ison MG, Kaul D, Michaels MG, et al. Considerations for screening live kidney donors for endemic infections: a viewpoint on the UNOS policy. Am J Transplant. 20I4;I4:IOO3-II. https:doi.org/IO.IIII/ajt.I2666

6. Kumar D, Humar A. Pandemic influenza and its implications for transplantation. Am J Transplant. 2006;6:1512-7.

7. World Health Organization. WHO Director-General's opening remarks at the media briefing on COVID-I9. 2020 [cited 2020 Apr 6]. Disponible en: https://www. who.int/dg/speeches/detail/who-director-general-sopening-remarks-at-the-media-briefing-on-covid-I9--II-march-2020

8. Li Q, Guan X, Wu P, Wang X, Zhou L, Tong Y, et al. Early transmission dynamics in Wuhan, China, of novel coronavirus-infected pneumonia. N Engl J
Med. 2020;382:II99-207. https:doi.org/IO.IO56/NEJMoa20oI316

9. Kakimoto K. Initial investigation of transmission of COVID-I9 among crew members during quarantine of a cruise ship - Yokohama, Japan, February 2020. MMWR Morb Mortal Wkly Rep. 2020 [cited 2020 Apr 6];69. Disponible en: https://www.cdc.gov/mmwr/volumes/69/wr/mm69iIe2.htm

Io. Worldometer. Colombia coronavirus cases. Woldometer. [cited 2020 Apr I2]. Disponible en: https://www. worldometers.info/coronavirus/country/colombia/

II. Husen P, Hornung J, Benko T, Klein C, Willuweit K, Buechter M, et al. Risk factors for high mortality on the liver transplant waiting list in times of organ shortage: A single-center analysis. Ann Transplant. 2019;24:2425I. https:doi.org/IO.I2659/AOT.9I4246

I2. Gadiparthi C, Cholankeril G, Yoo ER, Hu M, Wong RJ, Ahmed A. Waitlist outcomes in liver transplant candidates with high MELD and severe hepatic encephalopathy. Dig Dis Sci. 2018;63:1647-53. https:doi. org/I0.1007/sio62O-0I8-5032-5

I3. Clinical features of patients infected with 2019 novel coronavirus in Wuhan, China. [cited 2020 Mar 3I]. Lancet. 2020;395: 497-506. https:doi.org/Io.IOI6/SoI4O6736(20)30183-5

I4. World Health Organization. Novel coronavirus situation report 2 [Internet]. 2020 [cited 2020 Apr 6]. Disponible en: https://www.who.int/docs/default-source/ coronaviruse/situation-reports/20200I22-sitrep-2-20I9-ncov.pdf?sfvrsn=4d5bcbca_2

I5. World Health Organization. Open courses WHO. [cited 2020 Apr I2]. Disponible en: https://openwho.org/ courses

I6. Angelico R, Trapani S, Manzia TM, Lombardini L, Tisone G, Cardillo M. The COVID-I9 outbreak in Italy: initial implications for organ transplantation programs. Am J Transplant. 2020 Apr 3 [Online ahead of print]. https://doi.org/IO.IIII/ajt.I5904

I7. Kumar D, Manuel O, Natori Y, Egawa H, Grossi P, Han S-H, et al. COVID-I9: A global transplant perspective on successfully navigating a pandemic. Am J Transplant. 2020 Mar 23 [Online ahead of print]. https://doi. org/IO.IIII/ajt.I5876

I8. World Health Organization. Coronavirus disease (COVID-I9) technical guidance: Laboratory testing for 2019-nCoV in humans. [cited 2020 Apr I2]. Disponible en: https:/www.who.int/emergencies/diseases/ novel-coronavirus-20I9/technical-guidance/laboratory-guidance

19. Wang W, Xu Y, Gao R, Lu R, Han K, Wu G, et al. Detection of SARS-CoV-2 in different types of clinical specimens. Global Health. JAMA. Published online March II, 202O. https://doi.org/IO.IOOI/jama.2020.3786

20. Huang C, Wang Y, Li X, Ren L, Zhao J, Hu Y, et al. Clinical features of patients infected with 2019 no- 
vel coronavirus in Wuhan, China. Lancet Lond Engl. 2020;395:497-506. https://doi.org/IO.IOI6/SoI4O6736(20)30183-5

2I. Kumar D, Humar A. Emerging viral infections in transplant recipients. Curr Opin Infect Dis. 2005;I8:337-4I. https://doi.org/IO.IO97/oI.qco.0000I72697.44784.ff

22. Hamming I, Timens W, Bulthuis MLC, Lely AT, Navis GJ, van Goor H. Tissue distribution of ACE2 protein, the functional receptor for SARS coronavirus. A first step in understanding SARS pathogenesis. J Pathol. 2004;203:63I-7. https://doi.org/IO.IO02/path.I570

23. Li Z, Yi Y, Luo X, Xiong N, Liu Y, Li S, et al. Development and Clinical Application of a rapid IgM-IgG combined antibody test for SARS-CoV-2 infection diagnosis. J Med Virol. 2020 Feb 27 [Online ahead of print]. https:// doi.org/IO.IO02/jmv.25727

24. Aminian A, Safari S, Razeghian-Jahromi A, Ghorbani M, Delaney CP. COVID-I9 outbreak and surgical practice: Unexpected fatality in perioperative period. Ann Surg. 2020 Mar 26 [Online ahead of print]. https://doi. org/I0.I097/SLA.000oooooooo03925

25. Italian National Transplant Center. Information for transplant programs regarding novel Coronavirus 2019. [cited 2020 Apr 12]. Disponible en: http://www.trapianti.salute.gov.it/trapianti/homeCnt.jsp

26. Wu Z, McGoogan JM. Characteristics of and important lessons from the coronavirus disease 2019 (COVID-I9) outbreak in China: Summary of a report of $723 \mathrm{I} 4$ cases from the chinese center for disease control and prevention. JAMA. 2020;323:I239-42. https://doi.org/IO.IOOI/ jama.2020.2648

27. Hui DS, Azhar EI, Kim Y-J, Memish ZA, Oh M-D, Zumla A. Middle east respiratory syndrome coronavirus: risk factors and determinants of primary, household, and nosocomial transmission. Lancet Infect Dis. 20I8;I8:e2I7-27. https://doi.org/Io.IOI6/SI4733099(I8)30127-0

28. Centers for Disease Control and Prevention. People who are at higher risk for severe illness. 2020 [cited 2020 Apr 12]. Disponible en: https://www.cdc.gov/coronavirus/20I9-ncov/need-extra-precautions/people-athigher-risk.html

29. Liverpool Drug Interactions Group. Interactions with Experiemental COVID-I9 Therapies. Disponible en: https://www.covidı9-druginteractions.org/

30. Cao B, Wang Y, Wen D, Liu W, Wang J, Fan G, et al. A Trial of Lopinavir-Ritonavir in Adults Hospitalized with Severe Covid-I9. N Engl J Med. 2020 Mar I8. https://doi.org/IO.IO56/NEJMoa200I282

31. Gautret P, Lagier J-C, Parola P, Hoang VT, Meddeb L, Mailhe $\mathrm{M}$, et al. Hydroxychloroquine and azithromycin as a treatment of COVID-I9: results of an open-label non-randomized clinical trial. Int J Antimicrob Agents. 2020 Mar 20 [Online ahead of print]. https://doi.org/IO.IoI6/j.ijantimicag.2020.IO5949 